\title{
Qualidade de vida no trabalho dos Técnicos Administrativos do IFPB/Campus Patos
}

\author{
Quality of life on the work of Administrative Technicians of IFPB/Campus Patos
}

Calidad de vida en el trabajo de los Técnicos Administrativos del IFPB/Campus Patos

Recebido: 12/04/2021 | Revisado: 25/04/2021 | Aceito: 28/04/2021 | Publicado: 13/05/2021

\author{
Bárbara Birney Silva Dantas \\ ORCID: https://orcid.org/0000-0002-4961-3305 \\ Universidade Federal de Campina Grande, Brasil \\ E-mail: barbarabirney@gmail.com \\ Erivaldo Moreira Barbosa \\ ORCID: https://orcid.org/0000-0001-7479-1827 \\ Universidade Federal de Campina Grande, Brasil \\ E-mail: erifat@terra.com.br \\ Maria de Fátima Nóbrega Barbosa \\ ORCID: https://orcid.org/0000-0003-3415-8829 \\ Universidade Federal de Campina Grande, Brasil \\ E-mail: mfnobregabarbosa@gmail.com
}

\begin{abstract}
Resumo
A Qualidade de Vida no Trabalho (QVT) vem ganhando importância nas organizações modernas e instituições públicas, desde a década de 1990. O objetivo deste estudo é diagnosticar a percepção sobre QVT dos Técnicos Administrativos (TA's) do Campus Patos do Instituto Federal de Educação, Ciência e Tecnologia da Paraíba (IFPB) e descrever seu sentindo para subsidiar a proposição de ações e melhorias para sua qualidade de vida no trabalho. Esta pesquisa caracteriza-se como um estudo de caso de natureza quantitativa, exploratória e descritiva. Aplicou-se um questionário constituído por dez itens com aspectos sociofuncionais (Gênero, Idade, Nível do Cargo, Nível Hierárquico, Faixa Salarial, Escolaridade, Número de Dependentes, Tempo de serviço, função gratificada e Filiação sindical) adaptado do Modelo Biopsicossocial e Organizacional BPSO-96, de Limongi-França. Os resultados apontam que em relação à dimensão organizacional, os sujeitos apresentam bons índices de satisfação, sendo os mais baixos em relação à dimensão biológica. A avaliação geral aponta nível regular de satisfação, indicando a necessidade de avanços na área, bem como uma política institucional mais efetiva.
\end{abstract}

Palavras-chave: Qualidade de vida no trabalho; Percepção; Serviço público.

\begin{abstract}
Quality of Life at Work (QWL) has been gaining importance in modern organizations and public institutions since the 1990s. The aim of this study is to diagnose the perception of QWL of the Administrative Technicians (TA's) of the Campus Patos of the Federal Institute of Education, Science and Technology of Paraíba (IFPB) and describe their feelings to support the proposition of actions and improvements to their quality of life at work. This research is characterized as a case study of a quantitative, exploratory and descriptive nature. A questionnaire consisting of ten items with socio-functional aspects (Gender, Age, Job Level, Hierarchical Level, Salary Range, Schooling, Number of Dependents, Length of Service, Role and Syndical Membership) adapted from the Biopsychosocial and Organizational Model BPSO-96 from Limongi-France is applied. The results indicate that in relation to the organizational dimension, the subjects present good satisfaction rates, being the lowest in relation to the biological dimension. The overall assessment points to a regular level of satisfaction, indicating the need for progress in the area, as well as a more effective institutional policy.
\end{abstract}

Keywords: Quality of life at work; Perception of the work environment; Public service.

\section{Resumen}

La Calidad de Vida en el Trabajo (CVT) ha ganado importancia en las organizaciones modernas e instituciones públicas, desde la década de 1990. El objetivo de este estudio es diagnosticar la percepción sobre la CVT de los Técnicos Administrativos (TAs) del Campus Patos del Instituto Federal de Educación, Ciencia y Tecnología de la Paraíba (IFPB) y describir sus sentimientos para apoyar la propuesta de acciones y mejoras para su calidad de vida en el trabajo. Esta investigación se caracteriza por ser un estudio de caso de carácter cuantitativo, exploratorio y descriptivo. Se aplicó un cuestionario compuesto por diez ítems con aspectos socio-funcionales (género, edad, nivel de trabajo, nivel jerárquico, rango salarial, educación, número de dependientes, antigüedad en el servicio, función de bonificación y afiliación sindical) adaptado del Modelo Biopsicosocial y Organizacional BPSO-96, de LimongiFrance. Los resultados muestran que, en relación a la dimensión organizacional, los sujetos presentan buenos índices de satisfacción, siendo los más bajos en relación a la dimensión biológica. La valoración general apunta a un nivel 
regular de satisfacción, indicando la necesidad de avances en el área, así como una política institucional más efectiva.

Palabras clave: Calidad de vida en el trabajo; Percepción; Servicio público.

\section{Introdução}

A Lei 11.892, publicada em 29/12/2008, criou os Institutos Federais de Educação, Ciência e Tecnologia (IF's), que vem passando por uma expansão, objetivando uma atuação integrada e referenciada regionalmente. A finalidade dos IF's é promover a justiça social, a equidade, o desenvolvimento sustentável com vistas à inclusão social, bem como a busca de soluções técnicas e criação de novas tecnologias respondendo rapidamente e eficientemente às necessidades crescentes por formação profissional, difusão de conhecimentos científicos e de suporte à produção local, podendo atuar em todos os níveis e modalidades da educação profissional.

A proposta de retomada do crescimento econômico do país e a aplicação de ciência e tecnologia objetivando um trabalho produtivo e qualificado são fatores que demonstram a importância da formação escolar e profissional em todos os níveis. Nesse contexto é possível argumentar que a discussão sobre a percepção e satisfação da QVT assume vital importância para a manutenção dos institutos federais, trazendo ainda mais esse desafio para a educação, que não pretende, isoladamente, resolver todos os seus problemas, mas que, juntamente com um conjunto de ações e estudos multidisciplinares, pode vir a contribuir para a educação brasileira e os profissionais que nela atuam.

Na administração pública e no ambiente educacional, a falta de uma cultura de prevenção, a desvalorização da carreira do magistério e dos profissionais da educação, a falta de informação, resistência quanto ao uso de ferramentas de gestão, e a necessidade de implantação de políticas públicas de QVT para estes profissionais, são fatores que precisam ser superados. Assim é que os estudos ganham força na esfera pública, refletindo a necessidade fatual de gestão dessa área para que o bemestar dos servidores públicos seja também o da coletividade.

A qualidade de vida no trabalho é um tema que se tem mostrando em evidência, a exemplo dos trabalhos desenvolvidos por Limongi-França, Andrade e Stefano (2017), Limongi-França, Veloso e Schirrmeister (2007), Fell e Martins (2015), Sampaio (2012) e Oliveira, Silva, Castro e Limongi-França (2015). No que tange a qualidade de vida no serviço público alguns estudos correlatos podem ser evidenviados, como os estudos desenvolvidas por Ferreira, Alves e Tostes (2009), Damasceno e Alexandre (2012), Garcia (2007), Garcia (2017), Klein, Lemos, Pereira e Beltrame (2017), Nascimento (2016) e Verga e Valter (2016). Esses estudos reforçam a importância do tema nos mais diversos setores de atuação da atividade humana, em especial no serviço público.

Dessa maneira, partindo dos insights apresentados pelos estudos ora citados, o objetivo desse artigo é analisar como os servidores do IFPB - Campus Patos percebem a qualidade de vida em seu ambiente de trabalho.

\section{Conceito e Histórico da QVT}

Embora não haja um conceito formalizado e definitivo de QVT existe o consenso de que ela se alinha ao bem-estar do trabalhador. Segundo Lopes, Madruga, Avila e Beuron (2018) suas definições vão desde cuidados médicos estabelecidos pela legislação de saúde e segurança até atividades de integração e lazer. Do ponto de vista da Organização Mundial da Saúde (OMS), a QVT pode ser compreendida como "um conceito amplo que abrange a complexidade do construto e inter-relaciona o meio ambiente com aspectos físicos, psicológicos, nível de independência, relações sociais e crenças pessoais" (Fleck, 2000, p. 34). Há ainda uma vertente voltada para a participação na tomada de decisão trazendo a QVT como uma forma de abordar as relações organizacionais, ou seja, um método de redesenho do trabalho que envolve tomada de decisão da equipe e um movimento para melhorar a eficácia organizacional. 
O que se observa, de fato, é o surgimento de um novo paradigma sobre a QVT que traz o foco no trabalhador, visando garantir sua qualidade de vida e não somente a qualidade dos produtos e serviços que são resultado do trabalho (Ferreira, Ferreira, Antloga, \& Bergamaschi, 2009), de maneira que a gestão da QVT promova intrinsicamente essa transformação cultural lenta e gradual na qual o investimento da organização destina-se ao seu capital humano por meio do reconhecimento da necessidade de mudança para qual se exige o envolvimento de todos os seus membros (Mônaco \& Guimarães, 2000).

De todas as definições acima expostas podemos retirar um aporte conceitual convergente para a compreensão da realidade do trabalho contemporâneo livre de amarras ideológicas opressoras e, sobretudo, o resgate das dimensões de qualidade de vida e de trabalho, a partir de uma lógica onde o homem é a centralidade e não as razões econômicas e seus instrumentos reificadores (Dourado \& Carvalho, 2006). Observa-se no Quadro 1 a evolução do conceito:

Quadro 1 - Evolução do Conceito de QVT.

\begin{tabular}{|c|c|c|}
\hline Período & $\begin{array}{l}\text { Concepções evolutivas da } \\
\text { QVT }\end{array}$ & Características ou visão \\
\hline 1959 a 1972 & $\begin{array}{c}\text { QVT como uma variável (1959 } \\
\text { a 1972) }\end{array}$ & $\begin{array}{l}\text { Reação do indivíduo ao trabalho. Investigava-se como melhorar a } \\
\text { qualidade de vida no trabalho para o indivíduo. }\end{array}$ \\
\hline 1969 a 1974 & $\begin{array}{l}\text { QVT como uma abordagem } \\
\qquad(1969 \text { a 1974) }\end{array}$ & $\begin{array}{l}\text { O foco era o indivíduo antes do resultado organizacional; mas, ao } \\
\text { mesmo tempo, buscava-se trazer melhorias tanto ao empregado } \\
\text { como à direção. }\end{array}$ \\
\hline 1972 a 1975 & $\begin{array}{l}\text { QVT como um método (1972 a } \\
1975)\end{array}$ & $\begin{array}{l}\text { Um conjunto de abordagens, métodos ou técnicas para melhorar o } \\
\text { ambiente de trabalho e tornar o trabalho mais produtivo e mais } \\
\text { satisfatório. QVT era vista como sinônimos de grupo autônomos de } \\
\text { trabalho, enriquecimento de cargos ou desenho de novas plantas } \\
\text { com integração social e técnica }\end{array}$ \\
\hline 1975 a 1980 & $\begin{array}{l}\text { QVT como um movimento } \\
\qquad(1975 \text { a 1980) }\end{array}$ & $\begin{array}{l}\text { Declaração ideológica sobre a natureza do trabalho e as relações dos } \\
\text { trabalhadores com a organização. Os termos "administração } \\
\text { participativa" e "democracia industrial" eram frequentemente ditos } \\
\text { como ideais do movimento de QVT. }\end{array}$ \\
\hline 1979 a 1982 & $\begin{array}{l}\text { QVT como tudo } \\
\text { (1979 a 1982) }\end{array}$ & $\begin{array}{l}\text { Como panaceia contra a competição estrangeira, problemas de } \\
\text { qualidade, baixas taxas de produtividade, problemas de queixa e } \\
\text { outros problemas organizacionais. }\end{array}$ \\
\hline $\begin{array}{l}1982 \text { à década de } \\
90\end{array}$ & QVT como nada & $\begin{array}{l}\text { No caso de alguns projetos de QVT fracassarem no futuro, não } \\
\text { passará de um "modismo" passageiro. }\end{array}$ \\
\hline $\begin{array}{l}\text { A partir da } \\
\text { década de } 90\end{array}$ & $\begin{array}{l}\text { QVT como modelo ou filosofia } \\
\text { de gestão }\end{array}$ & $\begin{array}{l}\text { A capacidade de administrar o conjunto de ações, incluindo } \\
\text { diagnóstico, implantação de melhorias e inovações gerenciais, } \\
\text { tecnológicas e estruturais no ambiente de trabalho alinhada e } \\
\text { construída na cultura organizacional, com prioridade absoluta para o } \\
\text { Bem-Estar das pessoas na organização. }\end{array}$ \\
\hline
\end{tabular}

Fonte: Andrade (2016).

Portanto é possível observar o caminho que a QVT vem percorrendo desde o seu surgimento: inicialmente foi considerada uma mera variável; ou seja, uma maneira de reagir ao trabalho dentro da relação que com ele se estabelece; depois foi promovida à classe de abordagem gerencial dentro da cooperação do trabalhador para com os resultados da empresa; mais adiante foi um método em si de melhoria da produtividade; também foi considerada um movimento pela participação democrática do trabalhador dentro das decisões de gestão; chegou a um conceito global amplo que abarca diversas formas de lidar com questões de bem-estar e representou muitas vezes para alguns setores, sobretudo o setor público, uma descrença 
(Paiva \& Couto, 2008). Atualmente vivenciamos a fase de sua retomada como modelo de gestão a ser aplicado nas organizações.

Ferreira (2015) recorda que reconhecer alguns traços que marcam as mudanças no mundo corporativo é importante para entender o cenário histórico no qual emerge o interesse pela QVT e como ela tem ocupado as agendas de dirigentes, gestores, trabalhadores e também de pesquisadores de diversos campos das ciências do trabalho e da saúde.

Nos séculos XVIII e XIX os meios de produção foram sistematizados com vistas ao aumento da produtividade, eficácia e eficiência, passou-se então a discutir questões como condições de trabalho cientificamente. Nos anos 1950 Eric trist e seus colaboradores do Tavistok Institute, em Londres, trataram do bem-estar do trabalhador. Na década seguinte, como uma resposta à crise do capitalismo após os anos 1970 houve a necessidade de se reestruturar ideológica e politicamente o capital. Assim pode-se perceber que o interesse em QVT emergiu por volta de 1967 e 1974 diante da preocupação da sociedade norteamericana com os efeitos do trabalho na saúde e no bem estar geral dos trabalhadores e visando a melhoria do desempenho das pessoas, havendo nesse cenário duas vertentes teóricas: a que trazia uma resposta aos exageros da exploração do trabalhador presentes no taylorismo-fordismo e a que fazia uma constatação da importância de fatores subjetivos na produtividade, iniciada bem anteriormente pelos estudos de Elton Mayo em Hawthorne, em meados dos anos 20 (Batista, Antunes, \& Iizuka, 2012).

A partir da segunda metade do século XX, na década de 1980, num contexto de grande concorrência internacional, decorrente da automação das empresas japonesas, o governo americano destinou subsídios a iniciativas de redução dos custos com o trabalho, para conseguir manter a competitividade. Assim, "desde a década de 1970 salários equânimes, segurança no emprego, fatores motivacionais, realização e participação nas decisões de seu próprio trabalho, são pontos centrais dos estudos de QVT” (Lopes et al., 2018, p. 174).

No Brasil, em 1994 foi criada a Associação Brasileira de Qualidade de Vida (ABQV), organização sem fins lucrativos, que objetiva estimular ações e programas de qualidade de vida nos ambientes corporativos. De acordo com Limongi-França (1996), no Brasil, a maioria dos programas e ações de QVT se originaram de Segurança e Saúde no Trabalho, por exemplo as Normas Regulamentadoras da Legislação de Segurança e Saúde foram consolidas em 1978, junto à Comissão Interna de Prevenção de Acidentes (CIPA), Programa de Prevenção de Riscos Ambientais (PPRA), Programa de Controle Médico e Saúde Ocupacional (PCMSO), dentre outros (Batista et al., 2012). No âmbito acadêmico Eda Fernandes, em 1996, foi a pioneira dos estudos sobre a QVT. A partir do seu trabalho e ao longo de 15 anos até 2011 as principais instituições de ensino superior, especificamente no âmbito dos cursos de Administração e Negócios vieram incluindo em sua grade a disciplina QVT (Batista et al., 2012).

Segundo Ferreira (2015) nesse processo constante de mudança nos contextos das organizações contemporâneas, resultante do processo de reestruturação da economia mundial, que, conforme foi visto, teve como período histórico de referência a década 1965-1975 e como exemplo de fatores o aumento brutal do preço do petróleo; aumento das taxas de juros, juntamente com a crise no modelo de acumulação capitalista após redução drástica das taxas de mais-valia de maneira que essa reestruturação produtiva foi se configurando por meio de uma sinergia entre agentes econômicos e governamentais com o objetivo de sintonizar as mudanças dos modelos de gestão e relações de trabalho com a introdução e uso intensivo da microeletrônica, a reformulação do aparato jurídico dos Estados nacionais e, principalmente, com medidas voltadas para liquidar com as barreiras comerciais.

\section{Metodologia}

Para se chegar aos fins pretendidos por esse trabalho adota-se a pesquisa qualitativa e quantitativa, conforme Creswell (2007). Como estratégia de investigação qualitativa se utiliza o estudo de caso, conforme Bardin (2011). A presente pesquisa 
pode ser caracterizada ainda como bibliográfica, exploratória, descritiva e de campo, de acordo com Vergara (2013) e Marconi e Lakatos (2010).

Fez-se a escolha da observação direta extensiva por meio de questionário aplicado pessoalmente para todos os técnicos-administrativos lotados no IFPB/Campus Patos. A elaboração do instrumento foi feita por meio de uma adaptação do questionário da pesquisa de doutorado da professora Ana Cristina Limongi-França.

O questionário objetivou especificamente: avaliar o grau de satisfação dos participantes em ações e programas de QVT promovidos pelo Campus Patos, identificar o conceito de QVT para os TA's do Campus Patos e conhecer o nível de QVT em que o Campus Patos se encontra, sendo acompanhado de carta detalhada com o objetivo da pesquisa e telefone para informações.

O questionário de roteiro fechado foi composto por dois tipos de escala Ordinal, uma com notas de 0 a 10 (11 notas) com o objetivo de facilitar a interpretação, com as categorias de medida "satisfação total" (escore 2), "satisfação parcial" (escore 1) e "insatisfação" (escore 0). Outra com escolha e colocação de ordem e importância. Também foram utilizadas escalas nominais: SIM/NÃO/NÃO SEI; assinalar eventos que ocorrem; preencher com palavras-chave.

A análise das notas obtidas nos resultados foi convencionada de acordo com o Quadro 2:

Quadro 2 - Classificação da satisfação de acordo com as notas da escala.

\begin{tabular}{|l|c|}
\hline \multicolumn{1}{|c|}{ Notas } & Grau de Satisfação \\
\hline Menor que 5,0 & Baixa \\
\hline De 5,0 a 6,9 & Regular \\
\hline De 7,0 a 7,9 & Moderada \\
\hline Maior ou Igual a 8,0 & Alta \\
\hline
\end{tabular}

Fonte: Autores.

A aplicação do instrumento ocorreu durante o mês de junho de 2019, no Campus Patos do IFPB e o critério para inclusão na pesquisa foi a disponibilidade em responder o instrumento de coleta. Os questionários foram entregues pessoalmente em formato impresso. Os dados foram analisados por meio do Statistical Package for Social Sciences 21 (SPSS). Realizou-se estatística descritiva com a obtenção de valores de frequência absoluta e relativa (média, desvio padrão e frequência) e análises de estatística inferencial. Os testes utilizados foram de comparação de médias (teste t, ANOVA, Correlação de Pearson e Exato de Fisher). O nível de significância utilizado nas decisões dos testes estatísticos foi de 5\% e o intervalo de confiança de $95 \%$.

\section{Resultados e Discussão}

A Tabela 1 apresenta o perfil dos sujeitos da pesquisa caracterizado pela idade, gênero, nível de classificação e cargo, tempo de serviço, escolaridade, Unidades Organizacionais (UORG) de lotação, nível hierárquico e número de dependentes: 
Tabela 1 - Perfil dos Sujeitos da Pesquisa.

\begin{tabular}{|c|c|c|c|c|c|}
\hline \multirow{2}{*}{ Nível de Classificação do Cargo } & \multicolumn{3}{|c|}{ Lotação nas UORGS } & \multirow{2}{*}{ Subtotal } & \multirow{2}{*}{ Total } \\
\hline & DG & DAPF & DDE & & \\
\hline \multicolumn{6}{|l|}{ Idade } \\
\hline De 24 a 29 anos & - & 2 & 4 & 6 & \\
\hline De 30 a 35 anos & 1 & 6 & 7 & 14 & \\
\hline De 36 a 41 anos & 1 & 4 & 1 & 6 & 30 \\
\hline De 42 a 47 anos & - & - & 2 & 2 & \\
\hline De 48 a 53 anos & - & 2 & - & 2 & \\
\hline \multicolumn{6}{|l|}{ Gênero } \\
\hline Feminino & - & 3 & 8 & 11 & \\
\hline Masculino & 2 & 11 & 6 & 19 & 30 \\
\hline \multicolumn{6}{|l|}{ Nível de Classificação do Cargo } \\
\hline E - Assistente Social & - & - & 1 & 1 & \\
\hline E-Bibliotecário-Documentalista & - & - & 1 & 1 & \\
\hline E-Administrador & - & 1 & - & 1 & \\
\hline E - Pedagogo & - & - & 2 & 2 & \\
\hline E - Técnico em Assuntos Educacionais & - & - & 1 & 1 & 10 \\
\hline E-Medico & - & - & 1 & 1 & \\
\hline E - Analista de Técnico da Informação & - & 1 & - & 1 & \\
\hline E - Psicólogo & - & - & 1 & 1 & \\
\hline E-Contador & - & 1 & - & 1 & \\
\hline D - Assistente em Administração & - & 6 & 1 & 7 & \\
\hline D - Tradutor Interprete de Linguagem Sinais & 1 & - & 1 & 2 & \\
\hline D - Técnico em Eletrotécnica & - & - & 1 & 1 & \\
\hline D - Técnico em Contabilidade & - & 1 & - & 1 & 17 \\
\hline D - Técnico de Laboratório Área & - & 2 & - & 2 & 11 \\
\hline D - Técnico em Segurança do Trabalho & - & - & 2 & 2 & \\
\hline D - Técnico de Tecnologia da Informação & 1 & - & - & 1 & \\
\hline D - Técnico em Estrada & - & - & 1 & 1 & \\
\hline $\mathrm{C}-$ Auxiliar de Biblioteca & - & - & 2 & 2 & \\
\hline $\mathrm{C}-$ Assistente de Laboratório & - & 1 & - & 1 & \\
\hline \multicolumn{6}{|l|}{ Nível Hierárquico } \\
\hline Direção & - & 1 & - & 1 & \\
\hline Coordenação & 2 & 9 & 3 & 14 & 30 \\
\hline Operacional & - & 4 & 11 & 15 & \\
\hline
\end{tabular}

\section{Número de Dependentes}

Nenhum

Um

Dois

Três

Quatro

$\begin{array}{rrrrr}- & 2 & 5 & \mathbf{7} & \\ - & 5 & 5 & \mathbf{1 0} & \\ 1 & 5 & 4 & \mathbf{1 0} & \mathbf{3 0} \\ 1 & 1 & - & \mathbf{2} & \\ - & 1 & - & \mathbf{1} & \end{array}$

\section{Faixa Salarial}

De R\$1.000,00 a R\$3.000,00

De R \$ 3.001,00 a R \$ 5.000,00

Acima de R\$5.000,00

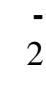

-

-
8
6

1

1

-

13

30

\section{Tempo de Serviço}

De 0 a 3 anos

De 4 a 7 anos

De 8 a 11 anos

$\begin{array}{rrrrr}1 & 3 & 7 & \mathbf{1 1} & \\ 1 & 10 & 5 & \mathbf{1 6} & \mathbf{3 0} \\ - & 2 & 1 & \mathbf{3} & \end{array}$

\section{Escolaridade}

Superior

Pós-Graduado

Mestrado

\begin{tabular}{rrrrr}
1 & 8 & 3 & $\mathbf{1 2}$ & \\
1 & 3 & 9 & $\mathbf{1 3}$ & $\mathbf{3 0}$ \\
- & 3 & 2 & $\mathbf{5}$ & \\
\hline
\end{tabular}


Tabela 1 - Perfil dos Sujeitos da Pesquisa.

\begin{tabular}{|c|c|c|c|c|c|}
\hline \multirow{2}{*}{ Nível de Classificação do Cargo } & \multicolumn{3}{|c|}{ Lotação nas UORGS } & \multirow{2}{*}{ Subtotal } & \multirow{2}{*}{ Total } \\
\hline & DG & DAPF & DDE & & \\
\hline \multicolumn{6}{|l|}{ Sindicalizados } \\
\hline Sim & 2 & 2 & 5 & 9 & \\
\hline Não & - & 12 & 9 & 21 & 50 \\
\hline Total por UORG & 2 & 13 & 15 & 30 & 30 \\
\hline
\end{tabular}

Legenda: Unidades Organizacionais (UORGs), Direção Geral (DG), Direção de Administração, Planejamento e Finanças (DAPF), Direção de Desenvolvimento do Ensino (DDE).

Fonte: Elaboração própria (2019) com base em dados da Coordenação de Gestão de Pessoas do Campus Patos.

Conforme observado na Tabela 3, as informações coletadas dos participantes permitiram elaborar um perfil, destacando-se:

a) o percentual mais expressivo, equivalente a 46\%, possui entre 30 a 35 anos de idade; e os menores percentuais, proporcionais a 6\%, estão igualmente nas faixas de 42 a 47 anos e 48 a 53 anos, ou seja, trata-se de uma amostra composta principalmente por adultos;

b) considerando apenas a idade, numa escala de idade máxima de 50 anos e uma mínima de 24 anos, apresenta-se uma média de 35,0 anos ( $\mathrm{DP}=6,57)$, sendo considerado, portanto, um público jovem;

c) em relação ao gênero, os participantes são 63,3\% do gênero masculino e o restante feminino, não tendo sido identificada nenhuma pessoa transgênero ou não binária;

d) quanto ao nível de classificação do cargo, a maior representatividade é de cargos de exigência de nível D (nível médio) com 60,0\%;

e) o cargo mais representativo na pesquisa é o de Assistente em Administração, cargo de atribuições mais genéricas, referindo-se a $23,3 \%$ dos cargos participantes;

f) no que se refere ao tempo de serviço, numa escala de tempo mínimo de 1 ano e máximo de 9 anos, a média é de 4,33 anos $(\mathrm{DP}=2,24)$, o que mostra que boa parte destes servidores é estável;

g) 43,3\% são pós-graduados;

h) A tríade das Diretorias Sistêmicas do Campus Patos, ou seja, DG, DDE e DAPF foi representada na pesquisa, sendo o número mais expressivo da Direção de Desenvolvimento do Ensino equivalente a 50\% dos participantes, contudo nota-se uma boa distribuição entre a DDE e a DAPF levando em consideração que a DAPF possui 43\%, sendo a representação menos expressiva na DG com $7 \%$;

i) Quanto ao nível hierárquico, percebe-se um equilíbrio entre o nível operacional e o das coordenações, tendo em vista que 50\% dos participantes atuam no nível operacional, 47\% atuam na Coordenação, recebendo para tanto função gratificada, e apenas um servidor possui cargo de Direção, representando apenas 3\% dos participantes, o que denota baixa representatividade quanto a tomada de decisão da gestão;

j) No que tange ao número de dependentes, a maioria possui entre 1 e 2 dependentes (33,3\%), sendo ainda razoável a quantidade de participantes que não possuem filhos;

k) Quanto à faixa salarial, 53,3\% dos participantes ganham entre R \$ 3.001,00 e R \$ 5.000,00 (três mil e um a cinco mil reais), ou seja, são servidores que recebem remuneração razoável ou mediana;

1) No que tange à filiação sindical, $70 \%$ dos participantes não são filiados ao sindicato dos servidores, o que levanta a suposição de não ser uma amostra que não se percebe unida enquanto categoria.

Destaca-se que a maioria relatou acreditar na pesquisa $(93,3 \%)$. 
$\mathrm{Na}$ análise do Bloco 1 do questionário, partiu-se de um somatório da escala dos resultados internos de satisfação da qualidade de vida no trabalho (QVT) e dividiu-se pelos números de questões, obtendo o seu total de escala por dimensão, ficando então a dimensão organizacional com média de 6,816 ( $\mathrm{DP}=1,44)$, a biológica com média 5,606 ( $\mathrm{DP}=1,79)$, a psicológica com média de 6,786 (DP=1,68), e a social com média de 5,211 (DP=1,90), conforme Gráfico 1 acima.

Gráfico 1 - Média de Satisfação por Dimensão.

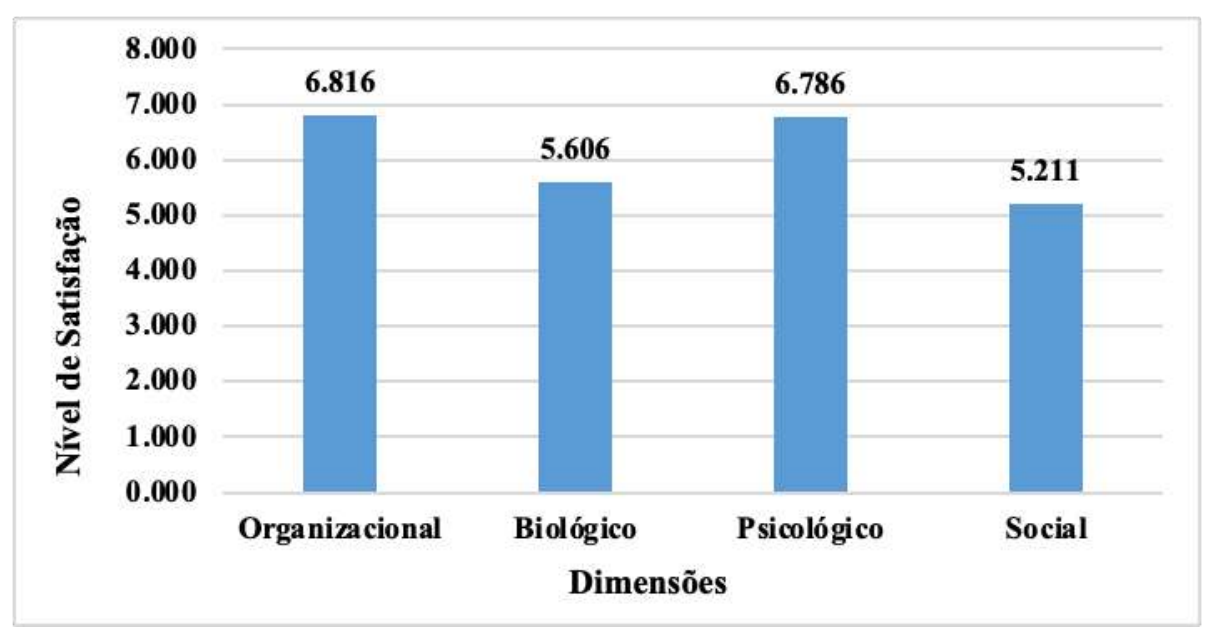

Fonte: Autores (2019).

Percebe-se, portanto que a dimensão organizacional foi a mais bem avaliada, não ficando a dimensão psicológica tão distante e sendo a dimensão social a que obteve a pior avaliação, contudo nenhuma das dimensões chegou a atingir o nível de satisfação nota 7,0 que equivaleria a um conceito "regular", embora esse trabalho não tenha utilizado essa linha conceitual, de maneira que pode-se afirmar que, em geral, a avaliação das dimensões tendem muito mais a uma posição de neutralidade do que de satisfação. Objetivando melhor destrinchar essa avaliação, realizou-se a análise descritiva dentro de cada dimensão, observando o comportamento de cada indicador que as compõem de acordo com a escala de satisfação do sujeito.

Dentro da dimensão organizacional há os seguintes indicadores e médias: Imagem da Instituição (Ima. Inst.) com média de 7,50, Desenvolvimento e treinamento (Desen.) com média de 7,13, Melhorias de Processo de Trabalho e Tecnologias (MPTT), média de 6,97, Participação da Tomada de Decisão (PTD), com média de 6,30, Redução da Burocracia (Red. Buroc.), com média 5,63, e Rotinas de Pessoal (Rot. Pess.) com média 7,33, demonstrados no Gráfico 2. 
Gráfico 2 - Dimensão Organizacional.

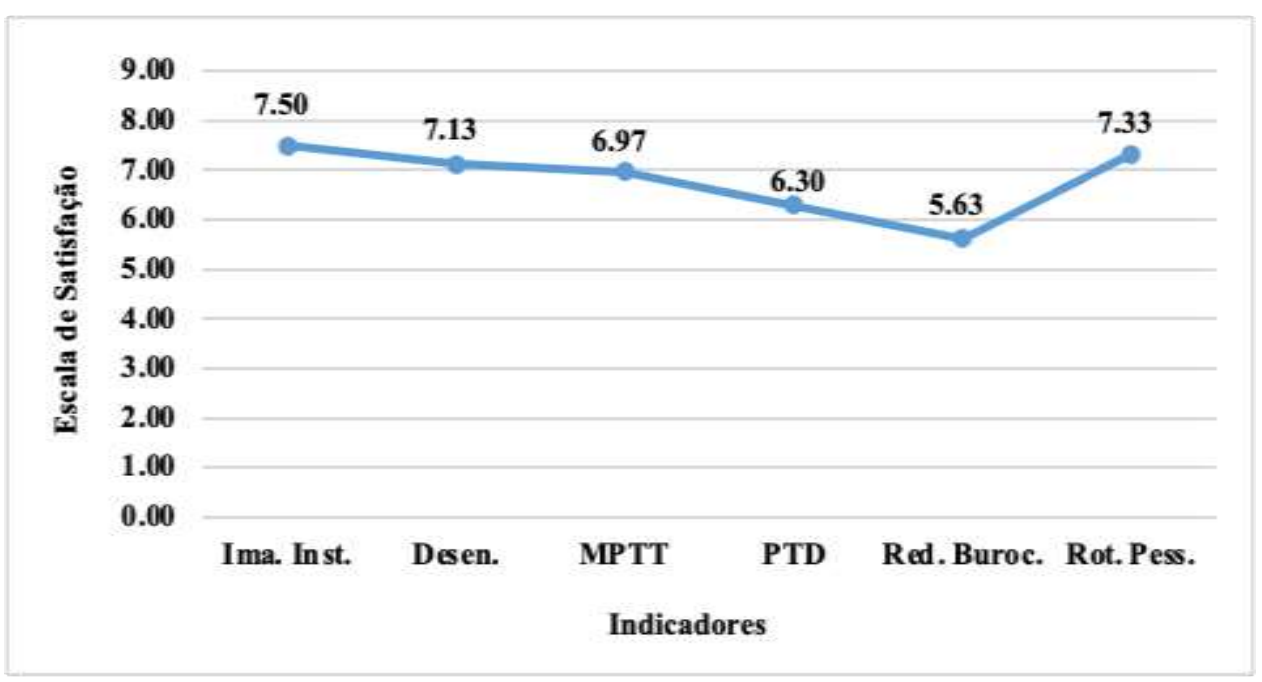

Fonte: Autores (2019).

Ou seja, o indicador melhor avaliado dentro dessa dimensão foi a imagem institucional e em seguida o atendimento das rotinas de pessoal, ultrapassando a nota de satisfação,7,0, o que corresponde a um nível de satisfação moderado, mais próximo do bom, refletindo uma avaliação positiva do atendimento da Coordenação de Gestão de Pessoas do Campus Patos e da área de gestão de pessoas em geral. Também houve uma boa média para o indicador de programas de treinamento e desenvolvimento, correspondentes à área de capacitação de servidores. Em contrapartida, o gráfico reflete ainda uma necessidade de redução da burocracia e melhoria do processo de participação na tomada de decisão.

Na sequência foram observados os indicadores da dimensão biológica no Gráfico 3:

Gráfico 3 - Dimensão Biológica.

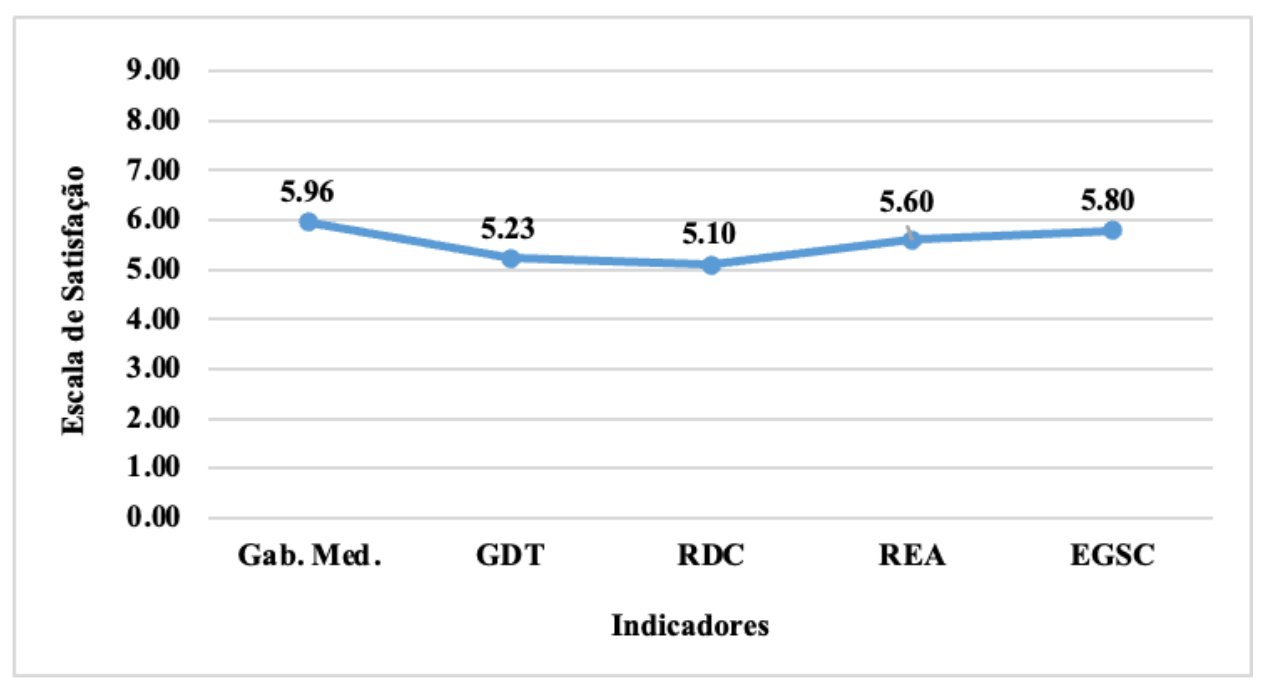

Fonte: Autores (2019).

Atendimento do gabinete médico (Gab.Med.) com média 5,96, Ginástica durante o trabalho (GDT), média 5,23, Refeições disponíveis no Campus (RDC:), com média 5,10, Riscos ergonômicos e ambientais (REA), apresentando media 5,60, e Estado Geral Saúde dos Colegas (EGSC), com média 5,80. 
Conforme se vê, a dimensão biológica possui um equilíbrio relativo de médias todas acima de 5,00 e abaixo de 6,00, o que por si só demonstra que, em comparação com as demais, não foi uma dimensão bem avaliada. Dentro dos seus indicadores as refeições disponíveis no Campus Patos foi o indicador com a pior avaliação, sendo seguida da oportunidade de realização de ginástica laboral. Nessa dimensão foi realizada uma Correlação de Pearson, para idade $(r=0,449 ; \mathrm{p}<0,05)$ que demonstrou significância, mostrando uma relação de possível influência da idade no nível de satisfação biológica, surpreendentemente os servidores mais velhos possuem maior satisfação com relação à dimensão biológica do que os mais novos, possivelmente porque a maior idade os proporciona parâmetros de comparação diferenciados.

A dimensão psicológica, possui os seguintes indicadores: Ferramentas de Avaliação de Desempenho (FAD), com média 6,50, Gestão do Clima Organizacional (GCO), com média 6,67, Plano de Carreira (Pla. Carr.), com média 6,47, Administrações de Salários (Adm. Sala.), com média 7,67, e Vida Pessoal dos Servidores (VPS), com média 6,63, demonstrados no Gráfico 4:

Gráfico 4 - Dimensão Psicológica.

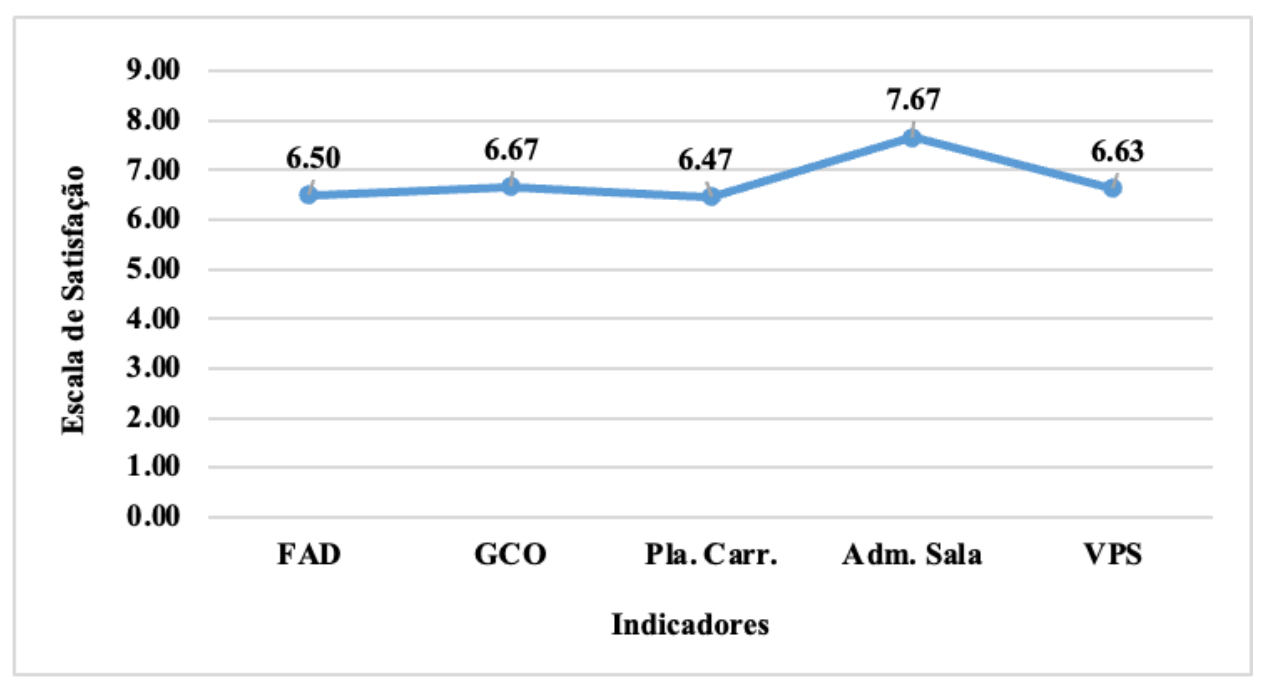

Fonte: Autores (2019).

A dimensão psicológica teve a melhor avaliação em comparação com as demais, sobretudo no indicador da administração de salários. Em contrapartida, o plano de carreira obteve a pior avaliação da dimensão, o que reflete uma luta sindical da categoria dos TA's já existente, qual seja a melhoria do PCCTAE. A ausência de interferência da vida pessoal dos servidores também foi mal avaliada, demonstrando a necessidade de maior separação entre o momento do trabalho e o momento da vida familiar/pessoal.

O Anova para variáveis não dicotômicas foi utilizado para analisar escolaridade $[F(2,27)=5,322 ; p<0,05]$ e nível de cargo [F $(2,27)=6,079 ; p<0,01]$ que apresentaram significância, ou seja, os participantes que têm Mestrado estão mais insatisfeitos. A possível causa requer investigação mais aprofundada e não é objetivo dessa pesquisa, contudo, existe a possibilidade desses servidores não estarem se sentindo devidamente aproveitados no que tange a sua titulação. Quanto ao nível do cargo percebe-se que os participantes que se encontram no nível hierárquico $\mathrm{D}$, que representam mais da metade da amostra, possuem satisfação mais elevada.

A dimensão social observou os seguintes indicadores: convênios comerciais (Conv. Comer.), com média 4,73, Atividades para Tempo Livre (ATL), com média 4,30, Atendimento aos Filhos (Atend. Filhos), com média 5,73, Valor do 
Auxílio-alimentação (VAuA), com média 4,70, Regime de Previdência (Reg. Prev.), com média 5,93 e Financiamento da Educação (Finan. Edu.), com média 5,43, como mostra a Gráfico 5.

Gráfico 5 - Dimensão Social.

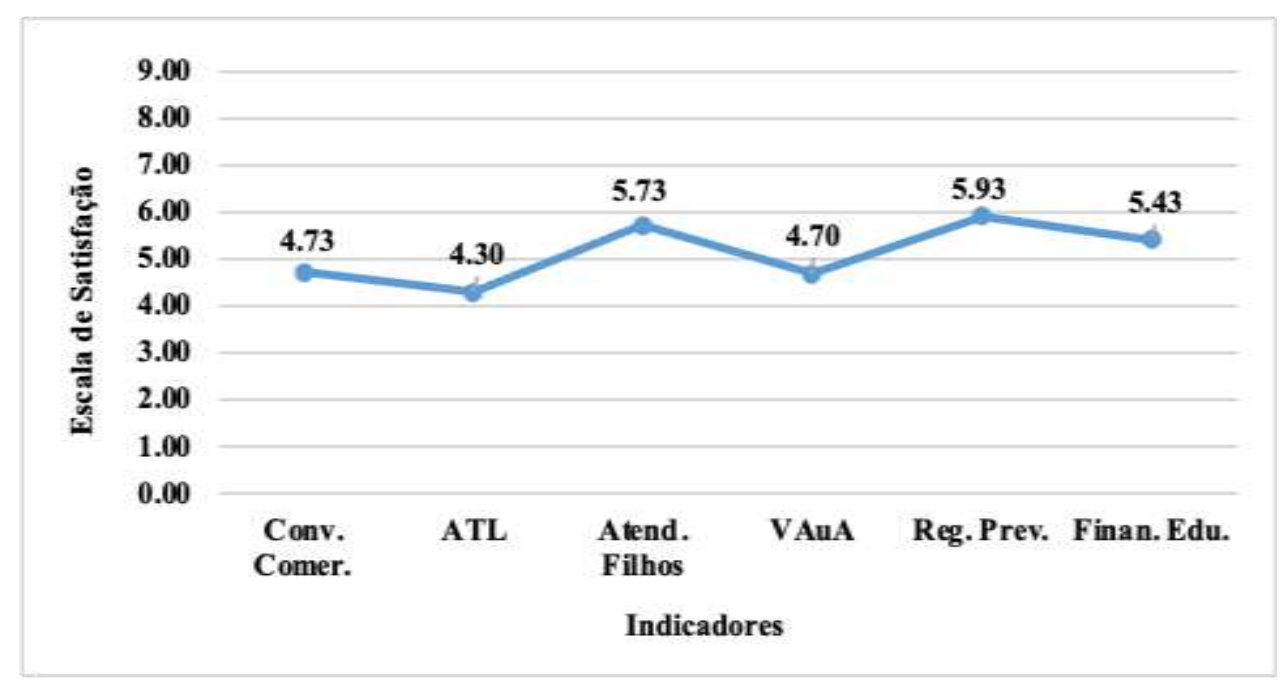

Fonte: Autores (2019).

A dimensão social, foi a dimensão que obteve a pior avaliação no geral, pois nenhum dos seus indicadores atingiu a média 6,0, tal e qual a dimensão biológica, porém com maior oscilação e médias consideravelmente menores, o que demonstra uma tendência à insatisfação, enquanto que na biológica a tendência foi à neutralidade. $\mathrm{O}$ indicador com a pior avaliação foi a atividade para tempo livre, o que denota que há pouca oportunidade para distração, lazer e momentos de vivência, carecendo, portanto, de maior integração. Além disso, o gráfico deixa claro também a insatisfação com o valor do auxílio-alimentação e a necessidade de se firmar mais e melhores parcerias e convênios comerciais para os servidores. Cruzando com os aspectos sociofuncionais. Foi utilizada a correlação para analisar a relação de idade ( $r=0,378 ; \mathrm{p}<0,05)$, significante. Assim, há uma relação da idade com a satisfação na dimensão social. O Anova foi utilizado para escolaridade $[F(2,27)=3,519 ; \mathrm{p}<0,05]$, que apresentou nível significante. Quanto a idade observa-se que os servidores mais novos estão mais insatisfeitos e os mais velhos mais satisfeitos. Possivelmente as expectativas criadas quando da entrada do serviço público podem vir a justificar esse dado. A mudança de mentalidade de uma geração para outra pode influenciar o que se espera nesse momento. No que tange à escolaridade, os servidores que possuem mestrado estão novamente mais insatisfeitos.

Quanto à percepção pessoal os indicadores analisados foram: Sensação de Bem-Estar no trabalho, Estado geral de tensão (stress) pessoal, Grau de satisfação com sua qualidade de vida, Adequação das ações de qualidade de vida do Campus Patos, Importância da Qualidade de Vida no trabalho para o resultado do trabalho, visando à percepção do sujeito de si próprio no decorrer do trabalho. Foi realizado um somatório da escala de satisfação usado no processo de coleta, e posteriormente, se atribuiu uma média geral para o estado pessoal, que foi de 6,50 ( $\mathrm{DP}=2,03)$, ou seja, um conceito regular de satisfação. Para variáveis não dicotômicas, foi utilizado o Anova, analisando assim, a faixa salarial $[F(2,27)=3,475 ; \mathrm{p}<0,05]$ que apresentou significância, ou seja, surpreendentemente o grupo que tem a faixa salarial mais alta está mais insatisfeito.

$\mathrm{Na}$ sequência dos fatos, analisou-se o bloco 2, buscando de fato a formação de um conceito. Foi então, somado o número de escolhas dos participantes por palavra, para ter uma média geral, tendo como maiores médias, Humanismo com 0 , $1778(\mathrm{DP}=0,16)$ e Realização Pessoal com 0, 1778 (DP= 0,16), como mostram os Gráfico 6 e 7. 
Gráfico 6 - Média do Conceito de QVT.

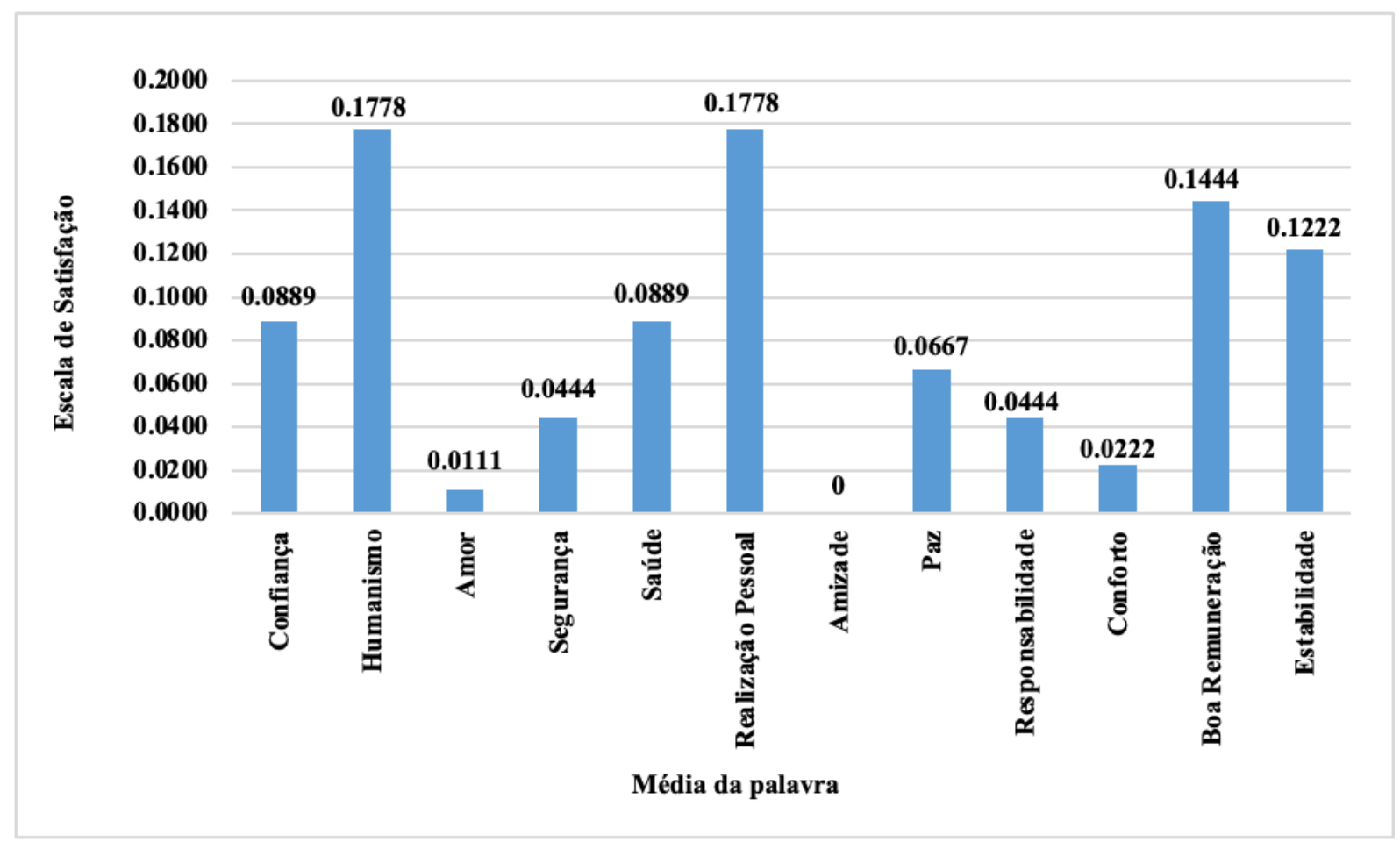

Fonte: Autores (2019).

Gráfico 7 - Médias do Nível de Importância.

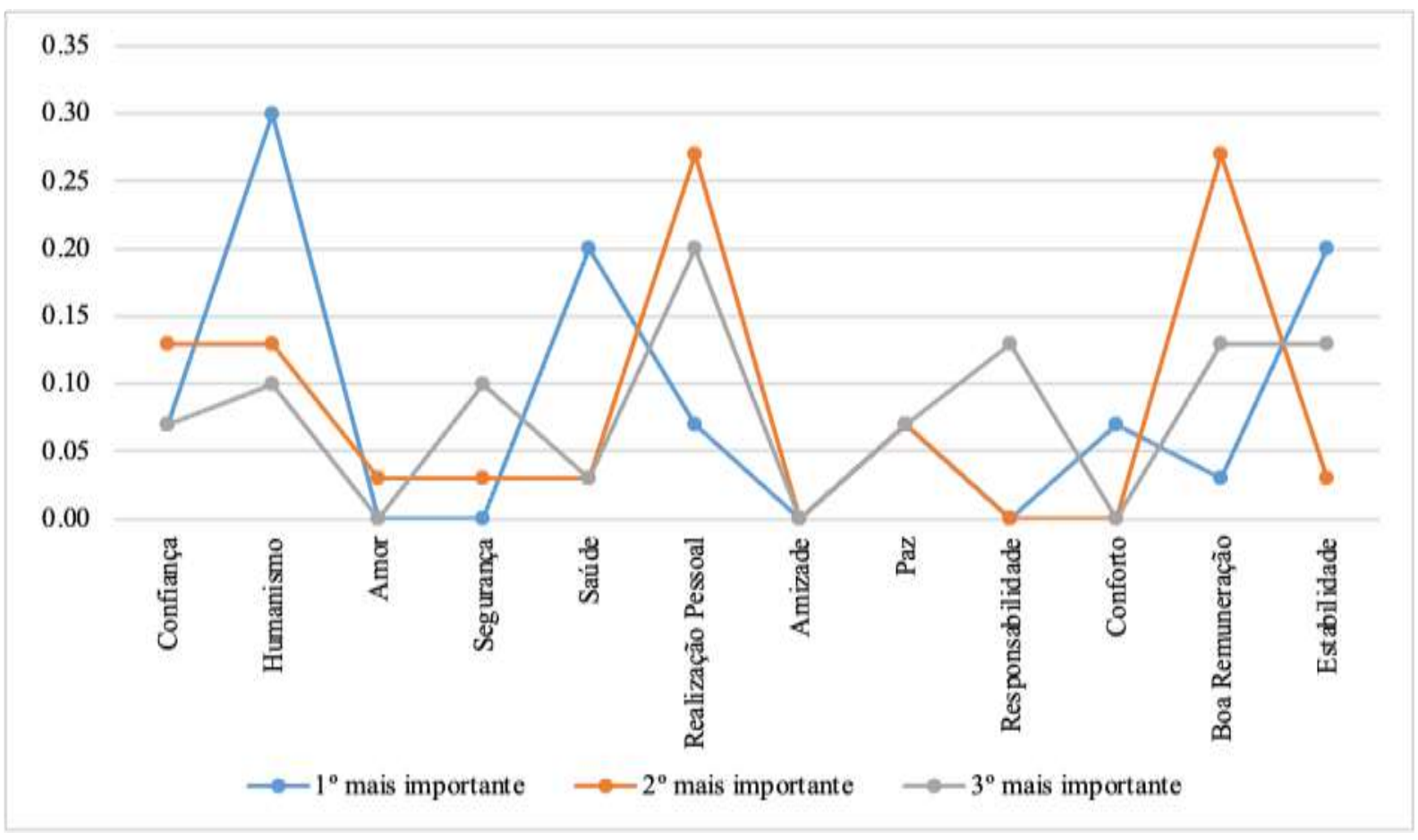

Fonte: Autores (2019).

As mesmas palavras foram analisadas de acordo com a ordem de relevância para os participantes, trazendo assim uma análise mais fiel ao possível conceito. Portanto, de acordo com o nível de importância, em primeiro lugar vem Humanismo 
com média 0,30 ( $\mathrm{DP}=0,46)$, em segundo lugar vêm Realização pessoal e Boa Remuneração empatadas com médias iguais de 0,27 ( $\mathrm{DP}=0,45)$, e em terceiro lugar vem Realização Pessoal com 0,20 (DP= 0,407).

A palavra mais citada, qual seja, humanismo, se relaciona com a dimensão organizacional, já a realização pessoal se alinha mais à dimensão psicológica. Interessante observar que houve um consenso quanto ao fator de ordem financeira como a boa remuneração ser considerado tão importante quanto a realização pessoal. Daí se pode inferir que esse grupo de servidores não busca apenas a estabilidade financeira e o conforto, mas que valorizam a dignidade, as capacidades e aspirações humanas, ou seja, se enxergam como agentes de uma cultura de desenvolvimento das potencialidades humanas e sua capacidade de transformação da social.

Os achados dessa pesquisa corroboram com as pesquisas desenvolvidas por Ferreira, Alves e Tostes (2009), Damasceno e Alexandre (2012), Garcia (2007), Garcia (2017), Klein, Lemos, Pereira e Beltrame (2017), Nascimento (2016) e Verga e Valter (2016), uma vez que tais estudos reforçam, como este, a importância do tema nos mais diversos setores de atuação da atividade humana, em especial no serviço público, contribuindo dessa forma para o desenvolvimento desse focus de pesquisa.

\section{Conclusão}

Este estudo descreveu a percepção dos técnicos administrativos do IFPB quanto à sua Qualidade de Vida no Trabalho. Dessa forma, destacam-se as seguintes conclusões sobre a percepção dos aspectos biopsicossociais: A nota geral foi 6,075 o que implica satisfação regular. A maior insatisfação ficou com a dimensão social (média 5,211), possuindo todas as suas notas abaixo de 5,0. A dimensão organizacional (média 6,816), entretanto, apresentou o maior nível médio de, tendo a imagem institucional e o atendimento às rotinas de pessoas como os itens mais bem avaliados. Não houve atribuição de notas acima de 8,0 (alta satisfação) em nenhum momento. Dentro da dimensão biológica (média 5,606) a atuação do gabinete médico foi o aspecto mais bem avaliado e as refeições disponíveis no Campus Patos o mais mal avaliado. Na dimensão psicológica (média 6,786) a administração de salários foi o aspecto mais bem avaliado e o plano de carreira o mais mal avaliado. Os cruzamentos com os aspectos sociofuncionais (Gênero, Idade, Nível do Cargo, Nível Hierárquico, Faixa Salarial, Escolaridade, Número de Dependentes, Tempo de serviço, função gratificada e Filiação sindical), não se demonstraram significativo na maioria dos casos. No que tange a percepção pessoal do estado de QVT surpreendentemente os dados apontam que grupo que tem a faixa salarial mais alta está mais insatisfeito, levando a concluir que para o grupo pesquisado a QVT não se relaciona necessariamente com retorno financeiro, o que é corroborado pelo conceito por ele formulado que traz humanismo como núcleo central.

Quanto às sugestões para novas pesquisas destaca-se: a realização da análise biopsicossocial com os docentes do Campus Patos e o desenvolvimento de um estudo comparativo entre a QVT dos técnicos administrativos e a dos docentes, pois, embora integrantes da mesma instituição, constituem carreiras distintas.

\section{Referências}

Andrade, S. M. (2016). Qualidade de Vida no Trabalho: proposta de um modelo integrador do BPSO com justiça organizacional para o bem-estar de servidores públicos. Tese de Doutorado. Faculdade de Economia, Administração e Contabilidade da Universidade de São Paulo - FEA-USP, São Paulo.

Bardin, L. (2011). Análise de Conteúdo. Edições 70.

Batista, M. B., Antunes, L. C., \& Iizuka, E. S. (2012). Qualidade de Vida no Trabalho e a Produção Acadêmica: Visita aos Anais dos ENANPAD’s de 2001 a 2011. XXXVI. Encontro da ANPAD.

Creswell, J. W. (2007). Projeto de Pesquisa: métodos qualitativo, quantitativo e misto (2a ed.). Artmed.

Damasceno, T. N. F., \& Alexandre, J. W. C. (2012). A Qualidade de Vida no Trabalho no âmbito do Serviço Público: Conceitos e análises. Revista Científica da Faculdade Darcy Ribeiro, 3. 
Dourado, D. C. P., \& Carvalho, C. A. (2006). Controle do homem no trabalho ou qualidade de vida no trabalho? Cad. EBAPE.BR [online], 4(4), 01-15.

Fell, A. F. A., \& Martins, D. F. V. (2015). Memória sobre a qualidade de vida no trabalho (QVT): uma perspectiva crítica. Perspectivas em Gestão \& Conhecimento, 5(2), 35-48.

Ferreira, M., Alves, L., \& Tostes, N. (2009). Gestão de qualidade de vida no trabalho no serviço público federal: o descompasso entre problemas e práticas gerenciais. Psicologia: teoria e pesquisa, 25(3), 319-327.

Ferreira, M. C. (2015). Qualidade de vida no trabalho: do assistencialismo à promoção efetiva. Revista Laboreal, 11(2), 28-35.

Ferreira, R. R., Ferreira, M. C., Antloga, C. S., \& Bergamaschi, V. (2009). Concepção e Implantação de Um Programa de Qualidade de Vida no Trabalho no Setor Público: O Papel Estratégico dos Gestores. Revista de Administração da Universidade de São Paulo - RAUSP, 44(2), $147-157$.

Fleck, M. P. O. (2000). O instrumento de avaliação de qualidade de vida da Organização Mundial de Saúde (WHOQOL-100): características e perspectivas. Ciência \& Saúde Coletiva, 5(1), 33-38.

Garcia, E. O. P. (2007). Estudo de caso exploratório sobre Qualidade de Vida no Trabalho de funcionários públicos. Organizações em Contexto, 3(5), 9-31.

Garcia, Y. M. (2017). Qualidade de Vida no Trabalho segundo a percepção dos técnicos administrativos do Instituto Federal de Educação, Ciência e Tecnologia do Ceará (IFCE). Organizações em contexto, 13(26).

Klein, L. L., Lemos, R. B., Pereira, B. A. D., \& Beltrame, G. (2017). Qualidade de vida no serviço público: uma avaliação em uma instituição de ensino superior. REAd, 23, 317-344.

Limongi-França, A. C. (1996). Indicadores empresariais de qualidade de vida no trabalho: esforço empresarial e satisfação dos empregados no ambiente de manufaturas com certificação ISO 9000. Tese de Doutorado. Faculdade de Economia, Administração e Contabilidade da Universidade de São Paulo - FEAUSP. São Paulo.

Limongi-França, A. C., Andrade, S. M., \& Stefano, S. R (2017). Dimensões da qualidade de vida no trabalho e justiça organizacional: um estudo com servidores públicos municipais. In: EnANPAD.

Limongi-França, A. C., Veloso, E. F. R., \& Schirrmeister, R (2007). A influência da qualidade de vida no trabalho em situações de transição profissional: um estudo de caso sobre desligamento voluntário. Revista Administração em Diálogo - RAD, 9(1), 35-58.

Lopes, R. P. V., Madruga, L. R. R. G., Avila, L. V., \& Beuron, T. A. (2018). Qualidade de vida e bem-estar no trabalho: autopercepção dos servidores de uma universidade federal. HOLOS, 34(06).

Marconi, M. A., \& Lakatos, E. M. (2010). Fundamentos de Metodologia Científica. Editora Atlas.

Monaco, F. F., \& Guimarães, V. N. (2000). Gestão da Qualidade Total e Qualidade de Vida no Trabalho: o Caso da Gerência de Administração dos Correios. RAC, 4(3), 67-88

Nascimento, A. C. (2016). Análise sobre a qualidade de vida no trabalho dos servidores do instituto federal de Roraima campus boa vista centro. Revista de Administração de Roraima-UFRR, 6(2), 322-345.

Oliveira, R., Silva, I, Castro, D., \& Limongi-França, A. (2015). Qualidade de Vida no Trabalho (QVT): um estudo com professores dos Institutos Federais. Holos, 6, 432-447. doi:https://doi.org/10.15628/holos.2015.1726

Paiva, K. C. M., \& Couto J. H. (2008). Qualidade de vida e estresse gerencial "pós-choque de gestão": o caso da Copasa - MG. Revista de Administração Pública, 42(6), 1189-1211.

Sampaio, J. R. (2012). Qualidade de Vida no Trabalho: Perspectivas e Desafios Atuais. Revista Psicologia: Organizações e Trabalho, 12(1), 121-136.

Verga, E., \& Walter, B. E. P. (2016). Qualidade de Vida dos Servidores Técnico-Administrativos de uma Instituição Federal de Ensino: Uma Avaliação pelo Instrumento WHOQOL-bref. In: XL Enanpad.

Vergara, S. C. (2013). Projetos e Relatórios de Pesquisa em Administração (14a ed.). Atlas. 